\title{
Einstein-Podolsky-Rosen paradox without entanglement
}

\author{
Ryszard Horodecki ${ }^{1,}$, , Michał Horodecki ${ }^{1, * *}$ and Paweł Horodecki $2, * * *$ \\ ${ }^{1}$ Institute of Theoretical Physics and Astrophysics, University of Gdańsk, 80-952 Gdańsk, Poland, \\ ${ }^{2}$ Faculty of Applied Physics and Mathematics, Technical University of Gdańsk, 80-952 Gdańsk, Poland
}

We claim that the nonlocality without entanglement revealed quite recently by Bennett et al. quant-ph/9804053 should be rather interpreted as Einstein-Podolsky-Rosen paradox without entanglement. It would be true nonlocality without entanglement if one knew that quantum mechnics provides the best possible means for extracting information from physical system i.e. that it is "operationally complete".

Pacs Numbers: 03.65.Bz

Quite recently a very impressive effect called "nonlocality without entanglement" has been revealed [1] within information-theoretic framework. The name "nonlocality" suggests that one deals with version of the Bell theorem [2] without entanglement. The purpose of this note is to point out that the effect should be instead treated as a counterpart of the Einstein, Podolsky, and Rosen (EPR) paradox [3].

Consider first the original EPR and Bell arguments. Without going into details, one can say that EPR showed that if predictions of quantum mechanics (QM) are correct then there is a conflict between two statements:

i) Nature can be described in a local-realistic way.

ii) Quantum mechanics provides a complete description of nature.

Call the view i) local realism (LR) (its negation is roughly called nonlocality, see [4 in this context) and ii) completness of quantum mechanics (CQM). Then the EPR paradox can be written the form

$$
Q M \text { predictions } \Rightarrow \sim(L R \wedge C Q M) .
$$

EPR believed that LR is true, so they concluded that QM is incomplete. It was Bell, who showed (by means of his famous inequalities [2] that this conclusion is wrong: he found that if we accept QM predictions, LR does not apply. So Bell argument has the form

$$
Q M \text { predictions } \Rightarrow \sim L R
$$

We see that Bell argument is apparently stronger statement than the EPR paradox. This is compatible with the fact that Bell took into account more predictions of quantum mechanics than EPR: the latter authors needed only correlations coming from the same observables for both subsystems (they used momentum and position), while Bell employed also correlations between different observables.

Note that here one is able to verify where nonlocality enters the scene: both EPR and Bell used predictions of
QM for entangled states. So, one can imagine that the pair of particles aquire nonlocal properties in result of coupling which produced the entanglement.

Let us now turn to the effect of Bennett et al. [1] where entanglement was not used, and try to find why could we say about nonlocality in this case. The scheme is the following. There are three parties: Alice, Bob and Charlie. Alice and Bob are distantly located and are allowed to communicate classically. Charlie prepares one of nine product and orthogonal states of two spin-1 particles and send one particle to Alice and the other one to Bob. Bennett et al. [1] showed that for a certain set of Charlie states Alice and Bob cannot recover the state. So if we imagine that Charlie wanted to send to Alice and Bob one of nine messages represented by the states, Alice and Bob cannot read the message. However, since the states are orthogonal, if Alice and Bob were not spatially separated, they could measure the state and read the message without any error [5]. So one can argue that the information carried by the pairs is nonlocal: it cannot be read from local properties of the systems and correlations between these properties. One can say that "information theoretic local realism" (ILR) is violated. This is what would be the counterpart of the Bell theorem. However, careful reader noted that in fact we did not refute conjunction

\section{$Q M$ predictions $\wedge I L R$}

as it should be to obtain Bell type argument. Indeed, we tacitly assumed, that QM provides the best possible means for extraction of information on Charlie preparation from the Alice and Bob particles. Translating it into the language parallel to that of EPR, we could say we assumed "operational completness of QM" (OCQM). Now we come to formula exactly analogous to (1):

$$
Q M \text { predictions } \Rightarrow \sim(I L R \wedge O C Q M)
$$

However, since it is demonstrated using predictions of QM only for product states, there is even stronger reason for refuting OCQM rather than ILR, than in the case of the original EPR paradox: here it is hard to imagine, when and where the nonlocality enters.

In conclusion, we have argued that "nonlocality without entanglement" should be regarded as "EPR paradox without entanglement" which still awaits for Bell argument without entanglement to become true nonlocality without entanglement. The main point is that one does not know, how to show that QM provides us with the 
best possible tools for extracting information from the single system. To obtain the true counterpart of Bell argument, one should provide a proof for that. A possible way out could be the following: better way of extraction of information will probably cause violation of the no-cloning theorem [6]. This violation would produce superluminal communication via entangled pair of particles [6]. In this way we would obtain that operational incompletness reduces to sending superluminal signals, so we could replace CQM from the sentence (3) with statement that superluminal singnaling is impossible. The problem is that we did it using entanglement... So, it seems that it will be rather difficult to produce Bell argument without entanglement. Anyway, the Bennett et al. effect has in fact the status of EPR paradox without entanglement.

The authors would like to thank Marek Żukowski for numerous discussions on EPR paradox and Bell's theorem. M. H. and P. H. gratefully acknowledge the support from the Foundation for Polish Science.

* E-mail address: fizrh@univ.gda.pl

** E-mail address: michalh@iftia.univ.gda.pl

*** E-mail address: pawel@mifgate.mif.pg.gda.pl

[1] C. H. Bennett, D. DiVincenzo, Ch. Fuchs, T. Mor, E. Rains, P. Shor, J. Smolin, W. K. Wootters, Report No. quant-ph/9804053, Phys. Rev. A (1999).

[2] J. S. Bell, Physics (N. Y.) 1, 195 (1964).

[3] A. Einstein, B. Podolsky and N. Rosen Phys. Rev. 47, 777 (1935).

[4] N. D. Mermin, J. Am. Phys 66, 920 (1998); H. Stapp, J. Am. Phys 66, 924 (1998).

[5] The question whether global measurement can be more efficient than local measurements was first raised in A. Peres and W. K. Wooters Phys. Rev. Lett 66, 1119 (1991). See also S. Massar and S. Popescu, Phys. Rev. Lett. 74, 1259 (1995).

[6] D. Dieks, Phys. Lett. A 92, 271 (1982); W. K. Wooters and W. H. Żurek, Nature (London) 299, 802 (1982). 